\title{
AUSÊNCIA DE RELAÇÃO ENTRE O TEMPERAMENTO E O AUTORRELATO DE EMOÇÕES A RESPEITO DE HOMOSSEXUAIS
}

Thalita Alves Lopes, Universidade de Rio Verde, thalita_lopes7@ hotmail.com. Claudio Herbert Nina-e-Silva, Laboratório de Psicologia Anomalística e Neurociências, Universidade de Rio Verde.

\section{Recebido em: 27/03/2014 - Aprovado em: 30/06/2014 - Disponibilizado em: 30/07/2014}

Resumo: O presente trabalho teve como objetivo verificar se há relação entre o temperamento e a expressão das emoções a respeito de homossexuais. Participaram do estudo 19 estudantes universitários. Para a coleta de dados foram utilizadas a escala BIS/BAS de avaliação do temperamento e o questionário proposto por Pereira (2004) para avaliação de emoções em relação a homossexuais. Verificou-se que não houve relação entre o temperamento e o autorrelato de emoções sobre homossexuais. Os resultados foram discutidos em termos da Teoria da Sensibilidade ao Reforço de Gray.

Palavras-chave: temperamento, personalidade, homofobia, emoções, atitudes, BIS/BAS.

Abstract: This study aimed to verify whether there is relation between temperament and expression of emotions toward homosexuals. Study participants were 19 university students. BIS / BAS scale and questionnaire assessment of temperament proposed by Pereira (2004) for assessing emotions toward homosexuals were used for data collection. It was found that there is no relationship between temperament and expression of emotions in relation to homosexuals. The results were discussed in terms of the Reinforcement Sensitivity Theory of Gray.

Keywords: temperament, personality, homophobia, emotions, attitudes, BIS/BAS.

\section{Introdução}

A homofobia como atitude negativa em relação aos homossexuais tem sido um problema social no nosso país (COLIN, 1999; LACERDA; PEREIRA; CAMINO, 2002; MARINHO; MARQUES; ALMEIDA; MENEZES; GUERRA, 2004). A necessidade de garantir os direitos civis dos homossexuais impôs novas reflexões acerca dos papeis sexuais em nossa sociedade (SANTOS; WINTER; SALLES; LONGO; TEODORO, 2007).

Contudo, apesar das conquistas jurídicas, a atitude desfavorável em relação à homossexualidade ainda é muito evidente no Brasil (LACERDA; PEREIRA; CAMINO, 2002; MARINHO et al., 2004; SANTOS et al., 2007).

$$
\text { Morgan (1981) afirma que o }
$$

preconceito qualquer que seja, é adquirido principalmente por meio de outras pessoas. E depois de estabelecidos dificilmente consegue-se modificá-los, pois na maioria das vezes está fortemente apoiado em aspectos emocionais.

O termo preconceito sexual se refere a atitudes negativas diante a um individuo ou grupo devido a sua orientação sexual (COLIN, 1999; MARINHO et al., 2004; SANTOS et al., 2007). O termo mais conhecido é a homofobia que é uma palavra derivada do grego "homos", que tem o mesmo significado de "o mesmo", e "phobikos", que significa "ter medo elou aversão a". Portanto, termo homofobia é utilizado para definir o medo e a repulsa referente à homossexualidade e aos homossexuais (MARINHO et al., 2004; SANTOS et al., 2007).

Pereira (2004) realizou um estudo com uma amostra de 374 estudantes de teologia de ambos os sexos da Pontifícia Universidade 
Católica de Goiás, cujo objetivo foi analisar as explicações da homossexualidade e o preconceito contra homossexuais por parte dos estudantes. Os resultados encontrados revelaram que os estudantes de teologia católicos mostraram claramente uma representação social bipolar referente à natureza da homossexualidade, onde as explicações ético-morais e religiosas se opuseram às explicações psicossociais. Também foi constatado que os estudantes do sexo feminino, com idade mais elevada e com mais tempo de estudo teológico atribuíram as causas da homossexualidade as explicações psicossociais rejeitando as explicações éticomorais e religiosas (PEREIRA, 2004).

O temperamento é a base biológica da personalidade, manifestando-se desde a infância, apresentando relativa estabilidade ao longo da vida e influenciando processos cognitivos e, sobretudo, emocionais das pessoas (CARVER; WHITE, 1994; DIEGO; FIELD; HERNANDEZ-REIF, 2001; CORR, 2002; ITO; GUZZO, 2002; BALCONI; FALBO; BAMBRILLA, 2009; HARMONJONES; HARRIS, 2009; KLEIN; LINHARES, 2010).

Considerando-se que o componente afetivo é considerado como a parte mais estável e, portanto, mais difícil de ser modificada em uma atitude (RODRIGUES, 1971; MORGAN, 1980; KRÜGER, 1986; MYERS, 2000), torna-se relevante analisar a relação entre o temperamento e a emoção à homossexualidade.
Desse modo, devido às evidências de influência do temperamento na manifestação das emoções, o objetivo do presente estudo foi verificar se há relação entre o temperamento e o autorrelato de emoções a respeito de homossexuais.

\section{Materiais e Métodos}

\subsection{Participantes}

Participaram 19 acadêmicos da Universidade de Rio Verde: três homens e 16 mulheres.

\subsection{Instrumentos}

O temperamento dos participantes foi avaliado por meio da Escala BIS-BAS (CARVER; WHITE, 1994), traduzida e adaptada para a realidade brasileira por Portilho-Souza e Nina-e-Silva (2013). A escala BIS/BAS está baseada no modelo de personalidade desenvolvida pelo psicólogo britânico Jeffrey Gray, a chamada Teoria da Sensibilidade ao Reforço, segundo a qual haveria uma modulação neurobiológica nas reações individuais à exposição a estímulos reforçadores e aversivos (CORR, 2002).

A escala BIS/BAS é composta por 24 itens, sendo que o participante deve classificar cada um desses itens conforme uma escala do tipo Likert de quatro pontos: $1=$ muito verdadeiro para mim; $2=$ mais verdadeiro do que falso para mim; $3=$ mais falso do que verdadeiro para mim; 4=totalmente falso para mim. A escala avalia as dimensões de 
temperamento Ansiedade (predomínio do Sistema de Inibição Comportamental - BIS) e Impulsividade (predomínio do Sistema de Ativação Comportamental - BAS) de cada indivíduo em questão, de acordo com sua opção marcada na escala.

A escala para a verificação das emoções dos participantes em relação aos homossexuais foi adaptado do instrumento desenvolvido por Pereira (2004), contendo 15 questões de uma escala tipo Likert de 7 pontos, versando sobre o grau de sentimentos em relação ao convívio com uma pessoa homossexual e o nível de emoção positiva (Admiração, Satisfação e Felicidade) e de emoção negativa (Nojo, Raiva, Desprezo) experimentada em relação à evocação da imagem de pessoas homossexuais.

\subsection{Procedimentos}

Após a aprovação do Comitê de Ética em Pesquisa da Universidade de Rio Verde (Parecer 182/2011), a coleta de dados ocorreu nas salas de aula do curso de Psicologia. Os acadêmicos que concordaram em participar do estudo receberam o Termo de Consentimento Livre e Esclarecido (TCLE) para leitura e eventual assinatura. Depois da assinatura do TCLE, os instrumentos foram entregues aos voluntários para que eles pudessem respondê-lo. A coleta de dados teve duração aproximada de 20 minutos.

\subsection{Análise dos dados}

A fim de verificar a existência de relação entre os escores de temperamento da escala BIS/BAS e os escores dos instrumentos de avaliação da atitude em relação aos homossexuais, o coeficiente de correlação produto-momento de Pearson ( $r$ de Pearson). As análises estatísticas foram feitas através de programa Statistical Package for the Social Science - SPSS for Windows (Versão 19.0).

\section{Resultados e Discussão}

Após a análise dos escores padronizados $z$ da Escala BIS/BAS, classificou-se 10 participantes na categoria temperamento "ansioso" e 9 participantes na categoria temperamento "impulsivo".

De acordo com Carver e White (1994) o BIS está relacionando a dimensão do temperamento caracterizada pela ansiedade, relacionando-se a indivíduos mais sensíveis a qualquer tipo de punição do que ao reforço. Os indivíduos nos quais o BIS está com maior evidência podem ser considerados como introvertidos (ansiosos) que sempre estão em alerta ou preocupados com a possibilidade de algo ruim acontecer (CARVER; WHITE, 1994).

Por outro lado, de acordo com a descrição de Carver e White (1994), os indivíduos com temperamento "impulsivo" são mais sensíveis ao reforço do que à punição. Os indivíduos nos quais predomina o BAS são considerados extrovertidos e impulsivos, por serem pessoas que estão 
sempre em busca de reforçadores mesmo diante a possível exposição de estímulos aversivos (CARVER; WHITE 1994).

A partir da análise dos escores médios e a variância dos graus de emoções positivas em relação aos homossexuais sentidas pelos participantes distribuídos conforme o tipo de temperamento, verificou-se que não houve diferenças estatisticamente significativas entre os temperamentos "ansioso" e "impulsivo" no que diz respeito os escores médios de "admiração" $\quad(t=-1,08709, \quad p<0,05)$, "satisfação" $\quad(t=-0,68455, \quad p<0,05) \quad \mathrm{e}$ "felicidade" $(t=-0,63485, p<0,05)$.

Esses resultados estão em desacordo parcial com a previsão da literatura (CARVER; WHITE, 1994; DIEGO; FIELD; HERNANDEZ-REIF，2001; CORR，2002; BALCONI; FALBO; BAMBRILLA, 2009; HARMON-JONES; HARRIS, 2009; HARMON-JONES et al., 2010) segundo a qual as pessoas de temperamento "impulsivo" estariam mais propensas a demonstrar emoções positivas do que as pessoas de temperamento "ansioso".

De acordo com Carver e White (1994), o Sistema de Ativação Comportamental (BAS), cujo predomínio produz o temperamento "impulsivo", é particularmente sensível ao reforço positivo. Então, pode-se considerar que o BAS está relacionado à experiência de sentimentos positivos como, admiração, felicidade, esperança e prazer (DIEGO; FIELD; HERNANDEZ-REIF,
Os nossos resultados que indicaram escores médios de "admiração", "satisfação" e "felicidade" ligeiramente mais altos para os participantes "impulsivos" do que para os participantes "ansiosos" não corroboraram os relatos da literatura segundo a qual os escores médios de emoções positivas para as pessoas "impulsivas" deveriam ser significativamente mais altos do que para as pessoas "ansiosas" (CARVER; WHITE, 1994; DIEGO; FIELD; HERNANDEZ-REIF，2001; CORR，2002; BALCONI; FALBO; BAMBRILLA，2009; HARMON-JONES; HARRIS， 2009; HARMON-JONES et al., 2010).

Por meio da análise dos escores médios e variância dos graus de emoções negativas em relação aos homossexuais sentidas pelos participantes distribuídos conforme o tipo de temperamento, observou-se que não houve diferenças estatisticamente significativas entre os temperamentos "ansioso" e "impulsivo" no que concerne aos escores médios de "nojo" $(t=-1,27844, p<0,05)$, "raiva" $(t=-0,5268$, $p<0,05)$ e "desprezo" $(t=-1,14459, p<0,05)$.

Esses resultados estão novamente em desacordo com a previsão de literatura (CARVER; WHITE, 1994; DIEGO; FIELD; HERNANDEZ-REIF，2001; CORR，2002; BALCONI; FALBO; BAMBRILLA, 2009; HARMON-JONES; HARRIS， 2009; HARMON-JONES et al., 2010), conforme a qual as pessoas consideradas "ansiosas" deveriam apresentar escores médios de emoções negativas significativamente mais 2001; CORR, 2002). 
altos do que as pessoas consideradas "impulsivas".

Por sua vez, o cálculo da correlação ( $r$ de Pearson) entre os escores de BIS e de BAS e os escores dos graus de emoções positivas em relação aos homossexuais relatadas pelos participantes evidenciou moderada correlação positiva entre os escores de BAS e os valores de Admiração ( $r=0,236597)$ e de Felicidade $(r=0,235413)$. Por outro lado verificou-se a ocorrência de correlação negativa entre os escores de BIS e os valores de Admiração ( $r=$ 0,54647) e Felicidade $(r=0,48151)$. Praticamente, não houve correlação estatisticamente significativa entre Satisfação e BAS $(r=-0,0225)$ e BIS $(r=-0,07598)$.

Esses resultados estão de acordo com a literatura por que, como já foi relatado, o BAS está relacionado a emoções positivas (CARVER; WHITE, 1994; DIEGO; FIELD; HERNANDEZ-REIF, 2001; CORR, 2002).

Apenas a emoção "satisfação", a qual apresentou praticamente nenhuma correlação com os escores de BIS e de BAS, não pôde ser comparada aos resultados da literatura, de acordo com a qual, deveria haver correlação positiva com BAS e correlação negativa com BIS (CARVER; WHITE, 1994; DIEGO; FIELD; HERNANDEZ-REIF, 2001; CORR, 2002; BALCONI; FALBO; BAMBRILLA, 2009; HARMON-JONES; HARRIS, 2009; HARMON-JONES et al., 2010).

A correlação negativa entre os escores de BIS e os escores das emoções Admiração e Felicidade também está de acordo com os dados encontrados na literatura, pois o BIS está relacionado à característica de temperamento "ansioso" e, portanto, a uma menor sensibilidade às emoções positivas (CARVER; WHITE, 1994; DIEGO; FIELD; HERNANDEZ-REIF, 2001; CORR, 2002).

O cálculo da correlação ( $r$ de Pearson) entre os escores de BIS e de BAS e os escores dos graus de emoções negativas em relação aos homossexuais relatadas pelos participantes evidenciou que não houve correlação estatisticamente significativa entre os escores de BAS e as emoções: Nojo ( $r=-$ 0,09182) e Desprezo ( $r=0,012441)$. Mas, por outro lado, registrou-se correlação negativa entre os escores de BIS e as emoções Nojo $(r=-0,36738)$, Desprezo $(r=-0,44139)$ e Raiva ( $r=-0,31371)$.

Esses dados estão totalmente em desacordo com a literatura, a qual apresentava a previsão de que seria observada correlação positiva entre os escores de BIS e as emoções negativas (CARVER; WHITE, 1994; DIEGO; FIELD; HERNANDEZ-REIF, 2001; CORR, 2002; BALCONI; FALBO; BAMBRILLA, 2009; HARMON-JONES; HARRIS, 2009; HARMON-JONES et al., 2010). Além disso, também deveria ter sido observada correlação negativa entre os escores de BAS e as emoções negativas (CARVER; WHITE, 1994; DIEGO; FIELD; HERNANDEZ-REIF, 2001; CORR, 2002).

No estudo realizado por Pereira (2004), demonstrou-se que os estudantes considerados "preconceituosos flagrantes" 
apresentaram nível mais elevado de preconceito e expressando maior rejeição em relação à intimidade e sentem mais emoções negativas (Nojo, Raiva, Desprezo), do que emoções positivas (Admiração, Satisfação e Felicidade). Já os estudantes considerados "preconceituosos sutis" apresentaram um grau relativamente moderado de rejeição à proximidade com homossexuais, expressando poucas emoções negativas e nenhuma emoção positiva. Desse modo, os nossos achados referentes às emoções positivas e às emoções negativas em relação aos homossexuais não estão de acordo com os dados encontrados na pesquisa de Pereira (2004).

\section{Conclusão}

Verificou-se que não houve relação entre o temperamento e a expressão, na forma de autorrelato, de emoções desfavoráveis a respeito de homossexuais. Sugere-se a realização de novos estudos que comparem as emoções em relação aos homossexuais em função do temperamento que utilizem instrumentos mais sutis de avaliação das emoções.

\section{Referências Bibliográficas}

BALCONI, M.; FALBO, L.; BRAMBILLA, E. BIS/BAS responses to emotional cues: self report, autonomic measure and alpha band modulation. Personality and Individual Differences, 47(8), p.858-863, 2009.

CARVER, C.S.; WHITE, T.L. Behavioral inhibition, behavioral activation, and affective responses to impending reward and punishment: The BIS/BAS scales. Journal of Personality and Social Psychology, 67, p.319-333, 1994.

COLIN, S. Homossexualidade: uma história. Rio de Janeiro: Record, 1999.

CORR, P.J. J. A. Gray's reinforcement sensitivity theory: tests of the joint subsystems hypothesis of anxiety and impulsivity. Personality and Individual Differences, 33, p.511-532, 2002.

DIEGO, M. A.; FIELD, T.; HERNANDEZREIF, M. BIS/BAS scores are correlated with frontal EEG asymmetry in intrusive and withdrawn depressed mothers. Infant Mental Health Journal, 22, p.665-675, 2001.

HARMON-JONES, E.; GABLE, P. A.; PETERSON, C. K. The role of asymmetric frontal cortical activity in emotion-related phenomena: A review and update. Biological Psychology, 84, p.451-462, 2010.

HARMON-JONES, E.; HARRIS, C. R. Jealousy: Novel methods and neural correlates. Emotion, 9, p.113-117, 2009.

ITO, P. C. P.; GUZZO, R. S. L. Temperamento: Características e determinação genética. Psicologia: Reflexão e Crítica, 15(2), p.232-245, 2002.

KLEIN, V. C; LINHARES, M. B. M. Temperamento e Desenvolvimento da Criança: Revisão Sistemática da Literatura. Psicologia em Estudo, 15(4), p.312-323, 2010.

KRÜGER, H. Introdução à Psicologia Social. São Paulo: EPU, 1986.

LACERDA, M; PEREIRA, C.; CAMINO, L. Um estudo sobre as formas de preconceito contra homossexuais na perspectiva das representações sociais. Psicologia: Reflexão e Crítica, 15(1), p. 162-171, 2002.

MARINHO, C. A; MARQUES, E. F. M; ALMEIDA, D.R; MENEZES, A. R. B; 
GUERRA, V. M. Adaptação da escala de homofobia implícita e explícita ao contexto brasileiro. Paidéia, 14(29), p.371-379, 2004.

MORGAN, C. T. Introdução à Psicologia. São Paulo: Mc Graw-Hill, 1981.

PEREIRA, A. S. L. Representações sociais do homossexualismo e preconceito contra homossexuais. 98f. Dissertação (Mestrado). Universidade Católica de Goiás, 2004.

PORTILHO-SOUZA, E.; NINA-E-SILVA, C. H. Tradução e adaptação da escala BIS/BAS para aplicação em adultos brasileiros. Revista da Universidade Vale do Rio Verde, 11(2), p.470-476, 2013.

RODRIGUES, A. Psicologia Social.

Petrópolis - RJ: Editora Vozes, 1971.

SANTOS, E. C; WINTER, F. S; SALLES, F. S; LONGO, J. L; TEODORO, M. L. M. Contato interpessoal e crenças sobre homossexualidade: desenvolvimento de uma escala. Interação em Psicologia, 11(2), p.470-476, 2007. 